\title{
A pure extradural hemangioblastoma mimicking a dumbbell nerve sheath tumor in cervical spine: illustrative case
}

\author{
José Piquer-Belloch, MD, PhD, ${ }^{1,2}$ Ruben Rodríguez-Mena, MD, $\mathrm{PhD},{ }^{1,2}$ José Luis Llácer-Ortega, MD, PhD, ${ }^{1,2}$ \\ Pedro Riesgo-Suárez, MD, PhD, ${ }^{1,2}$ Vicente Rovira-Lillo, MD, PhD, ${ }^{1,2}$ Alain Flor-Goikoetxea Gamo, MD, ${ }^{1,2}$ \\ Antonio Cremades-Mira, MD, ${ }^{3}$ and Eva Llopis-San Juan, $\mathrm{MD}^{4}$
}

Departments of ${ }^{1}$ Neurosurgery, ${ }^{3}$ Pathology, and ${ }^{4}$ Radiology, Hospital Universitario de la Ribera, Alzira, Valencia, Spain; and ${ }^{2}$ Cátedra de Neurociencias-Fundación Vithas-CEU, Valencia, Spain

\begin{abstract}
BACKGROUND Spinal extradural hemangioblastomas (HBs) are quite uncommon, with most reported cases involving the thoracic and lumbar areas. Therefore, the presence of a dumbbell-shaped pure extradural cervical HB is exceptional, making preoperative diagnosis particularly challenging.

OBSERVATIONS The authors report a case of a 27-year-old woman who presented to their outpatient clinic with progressive cervicobrachialgia and numbness in the left arm. Magnetic resonance imaging showed a C5-6 intradural extramedullary lesion, and, despite some atypical features, the diagnosis of a possible neurogenic tumor was made. A multidetector computed tomography scan and angiography confirmed the expansion and remodeling of the left neural foramen as well as the highly vascularized nature of the mass. Preoperative embolization of the lesion was performed. Complete tumor resection was accomplished, followed by a C5-6 posterior fusion. Histopathology and immunohistochemistry revealed an HB.

LESSONS HBs should be considered among the differential diagnosis of cervical extradural tumors. Exhaustive preoperative workup and surgical planning are decisive in order to attain gross-total resection with favorable outcomes.
\end{abstract}

https://thejns.org/doi/abs/10.3171/CASE2192

KEYWORDS cervical; diagnosis; dumbbell; extradural; hemangioblastoma; surgery

Hemangioblastomas (HBs) are benign neoplasms of mesodermal or neuroectodermal origin that can appear anywhere in the human body. ${ }^{1-3}$ Central nervous system HBs (CNSHBs) may occur sporadically or in association with Von Hippel-Lindau (VHL) disease. Frequently, they are found in the intradural compartment of the posterior cranial fossa or spinal cord, in the latter, usually presenting as an intraextramedullary mass or even affecting a spinal nerve root. Most of the reported cases involve the thoracic and lumbar regions, but spinal extradural location is extremely rare. ${ }^{4}$ Cervical spine $\mathrm{HBs}$ are exceptional ${ }^{5-8}$ and, to our knowledge, a pure extradural dumbbell HB in this location has not been described previously.

Although it represents an imaging diagnostic challenge in which accurate recognition might not be possible, it is important to be aware of this rare entity when analyzing preoperative studies.
Conceiving the relation of the tumor with the surrounding vessels is of paramount significance in surgical planning in order to avoid massive intraoperative bleeding, which is critical when performing cervical spinal cord tumor surgery. Because of the limited reported cases, it is essential to identify and learn the principal imaging findings and biological behavior of cervical spinal extradural HBs and select the most appropriate surgical intervention.

\section{Illustrative Case}

A 27-year-old female presented with an 8-month complaint of left cervicobrachialgia with minor $\mathrm{C5}-6$ sensorimotor radiculopathy on physical examination. There were no remarkable findings in her medical history, and she denied a family history of central nervous

ABBREVIATIONS CNSHB = central nervous system hemangioblastoma; GDTA = gadolinium; GFAP = glial fibrillary acidic protein; HB = hemangioblastoma; $\mathrm{MRI}=$ magnetic resonance imaging; $\mathrm{MDCT}=$ multidetector computed tomography; $\mathrm{PVA}=$ polyvinyl alcohol; SI = signal intensity; T1Wi = T1-weighted image; $\mathrm{T} 2 \mathrm{Wi}=\mathrm{T} 2$-weighted image; VA = vertebral artery; VHL = Von Hippel-Lindau. INCLUDE WHEN CITING Published August 2, 2021; DOI: 10.3171/CASE2192. SUBMITTED February 17, 2021. ACCEPTED May 10, 2021.

(C) 2021 The authors, CC BY-NC-ND 4.0 (http://creativecommons.org/licenses/by-nc-nd/4.0/). 

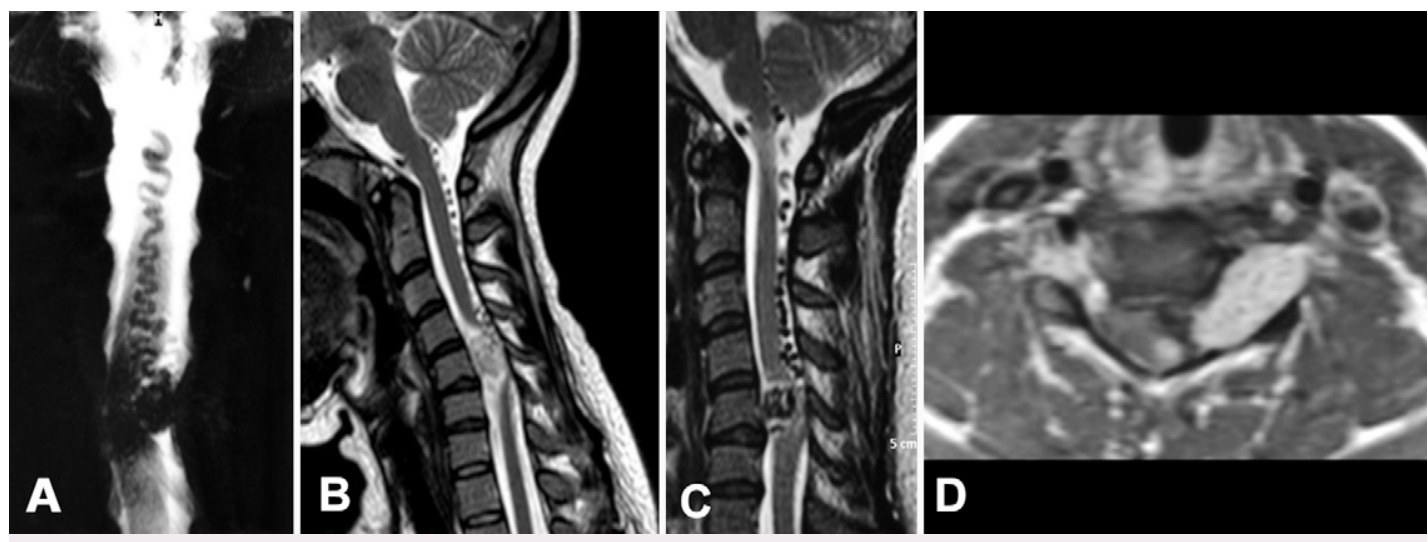

FIG. 1. Cervical spine MRI. Coronal gradient-echo image (A) and sagittal fast spin-echo (FSE) T2Wis (B and C) showing an extraintradural C5-6 tumor with high SI areas in the spinal cord due to compressive myelopathy. The mass shows heterogenous $\mathrm{SI}$, with signal voids of serpiginous shape related to dilated vascular channels associated to prominent drainage veins that ascend toward the brainstem. On axial FSE T1Wi (D), the lesion demonstrates intense and homogeneous enhancement after gadolinium contrast injection, extending into the left neural foramen.

system diseases. The laboratory data were all within the normal range. Magnetic resonance imaging (MRI) demonstrated a well-defined C5-6 apparent intradural extramedullary lesion, anterior to the spinal cord, expanding and extending through the left intervertebral foramen, and with posterolateral displacement and compression of the spinal cord toward the right side. The mass diameters were $21 \mathrm{~mm} \times 20 \mathrm{~mm} \times$ $29 \mathrm{~mm}$, showing low signal intensity (SI) on T1-weighted images (T1Wis), heterogeneous SI on T2-weighted images (T2Wis) with low SI dots, and strong and homogenous enhancement after gadolinium (GDTA) contrast intravenous injection. Signal void images where seen in the upper cervical spine on T2Wis representing prominent vessels within the cerebrospinal fluid (Fig. 1). A multidetector computed tomography (MDCT) scan with posterior multiplanar reconstructions displayed cervical spine incipient kyphotic deformity, widening of the left C5-6 intervertebral foramen with bone remodeling, invasion of transverse foramen, and partial destruction of the ipsilateral facet joint and pedicle, suggesting mechanical instability (Fig. 2). The main preoperative tumor diagnosis was a vascular schwannoma, which prompted us to carry out a spinal angiography, depicting a hypervascularized tumor, whose blood supply depended on the left vertebral and ascending and deep cervical arteries. Embolization of major feeding vessels was ac-
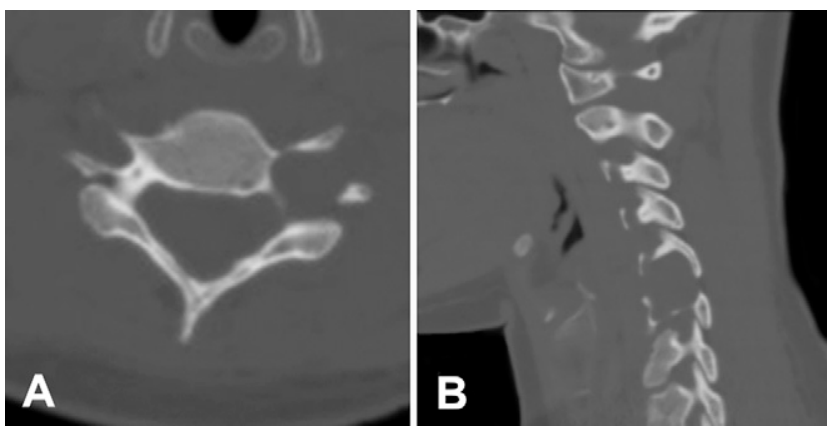

FIG. 2. MDCT of the cervical spine, axial plane, and multiplanar reconstruction on sagittal plane: widening of the left C5-6 neural foramen. Note the smooth contour of the edges indicating slow-growing mass (A and B). complished using spherical polyvinyl alcohol (PVA) particles of 150$300,300-500$, and 500-700 $\mu \mathrm{m}$ (Fig. 3).

Through a conventional posterior midline cervical approach, a C5-6 laminectomy with removal of the remaining left eroded facet was necessarily performed in order to expose a noticeably dumbbell-shaped totally extradural tumor, partially thrombosed due to previous embolization. En bloc excision of the tumor was accomplished using standard microsurgical techniques with minimal spinal cord manipulation, in conjunction with meticulous protection of vertebral artery (VA) and the exiting nerve root. After dural opening, there was no evidence of any intradural extension of the tumor. Finally a C3-7 posterior lateral mass screw fixation was carried out to promote posterolateral fusion (Fig. $4 \mathrm{C}$ and D).

Histopathology revealed a highly vascular neoformation, with hemangioma-type vessels of different sizes and other regions with higher cellularity ("stromal cells") containing microvacuolated lipidladen cytoplasm. The immunohistochemical profile included positivity to neuron-specific enolase and S-100 protein in the stromal cells, progesterone receptor focally, CD34 (endothelial cells); and negativity to glial fibrillary acidic protein (GFAP). The diagnosis of HB (World Health Organization Grade I) was then established (Fig. 5).

The postoperative course was uneventful, and the patient did not develop any worsening of her previous neurological deficits. MRI confirmed complete resection of the lesion (Fig. 4A and B). Genetic molecular testing for VHL disease was negative. Ophthalmic evaluation was normal, and chest, abdominal, and pelvic contrast MDCT did not reveal other tumors. She attended an outpatient physical therapy program, and, after approximately 7 months, she completely recovered from the paresthesias and weakness in the left upper limb. Afterward, the patient remained asymptomatic, and no tumor regrowth has been detected on serial control MRI over a 3-year follow-up period.

\section{Discussion}

\section{Observations}

CNSHBs more frequently present as posterior cranial fossa and/ or spinal cord intradural lesions. Extradural involvement is relatively infrequent and usually derived from tumor growth and extension from 


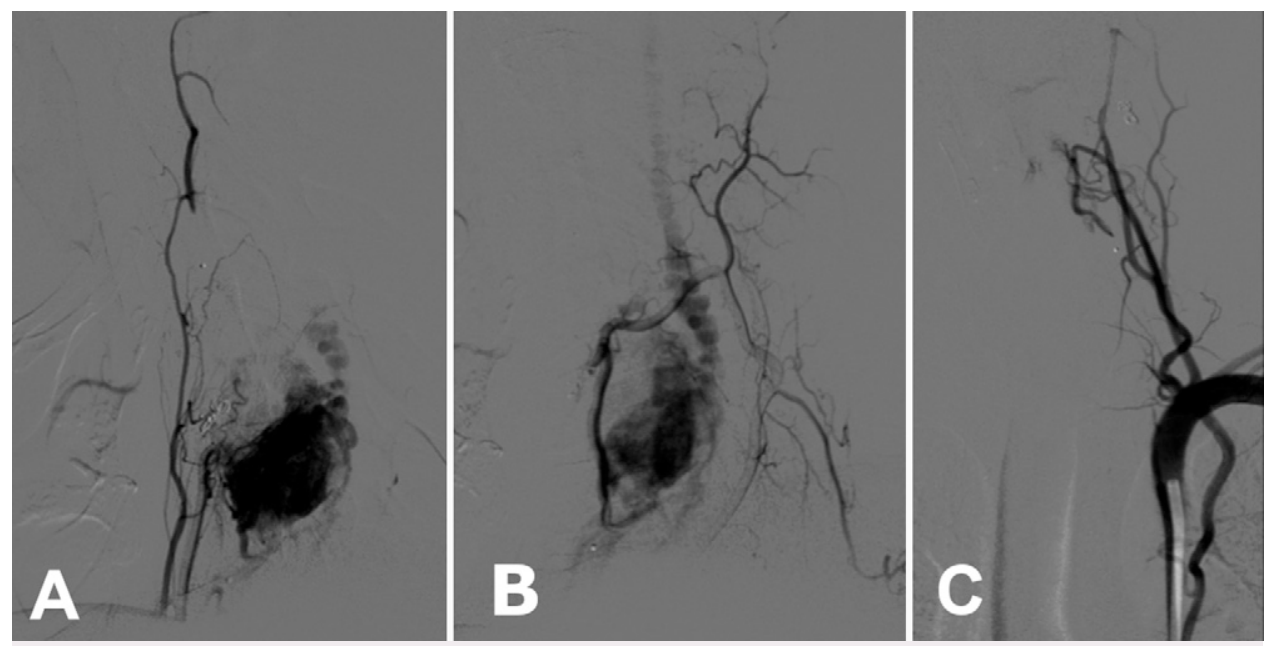

FIG. 3. Selective angiography and embolization of the tumor. We can appreciate the occlusion of the left VA; however, the vertebral segment feeding the tumor appears to be patent, showing collateral circulation coming from the ascending cervical $(\mathbf{A})$ and deep cervical $(\mathbf{B})$ arteries. The anterior spinal artery emerges from the VA as well as the left ascending cervical artery. Both arteries are catheterized and progressively embolized using PVA spherical particles measuring 150-300, 300-500, and 500-700 $\mu \mathrm{m}$. The final angiographic control shows successful complete devascularization of the lesion (C).

its intradural component. ${ }^{2,4,9}$ However, a pure extradural HB of the spinal nerve root is extremely rare, and association with VHL disease has been reported in up to $45 \%$ of the cases., ${ }^{3,7,10}$ Aytar et al. recently reviewed 21 studies that included 38 patients with pure extradural spinal $\mathrm{HBs},{ }^{4}$ and most of the cases (85.4\%) affected the thoracic or lumbar spine. Extradural cervical HBS are exceptional, with only five cases reported so far. ${ }^{5-8}$ Furthermore, there have been two cases of both intra and extradural dumbbell-shaped lesions found in the literature, ${ }^{1,11}$ but this is the first description of a complete extradural dumbbell-shaped cervical HB.

Consequently, the differential diagnosis of dumbbell-shaped cervical tumors presenting with intervertebral foramen enlargement should include schwannoma, meningioma, ependymoma, or metastasis but also nerve root HB with possible intradural extension. Most of the lesions will show low or iso-SI on T1Wis and high SI on T2Wis, with homogeneous enhancement after GDTA injection. ${ }^{12}$ Widening of the spinal foramen is nonspecific and could be associated with either meningeal or neurogenic tumors; for that reason, it should not be used as a reliable MRI sign. On T2Wis, the presence of prominent vessels in the subarachnoid space together with low $\mathrm{SI}$ tumor areas are key features in the diagnosis of these neoplasms. ${ }^{4,12}$ MDCT might show bone destruction or erosion, as well as augmented interpedicular distance, which are useful for the evaluation of further spinal instability.

The surgical challenges of this kind of cervical spine tumors are determined by three main aspects: selection of a safe approach, achievement of complete resection, and stabilization of the spine. It is essential to evaluate tumor extension to the adjacent structures, including bone, nerve root, and VA, and biomechanical considerations of the spine increase technical complexities in the surgical approach. A posterior approach with laminectomy and unilateral facetectomy has been proposed for dumbbell cervical tumors. ${ }^{10,13}$ Other authors postulate in favor of a lateral approach, ${ }^{11}$ which, on the other hand, would bring the VA to sight before having access to the lesion, adding difficulties in accomplishing a safe complete excision. In our case, removal of the residual bone from the impaired
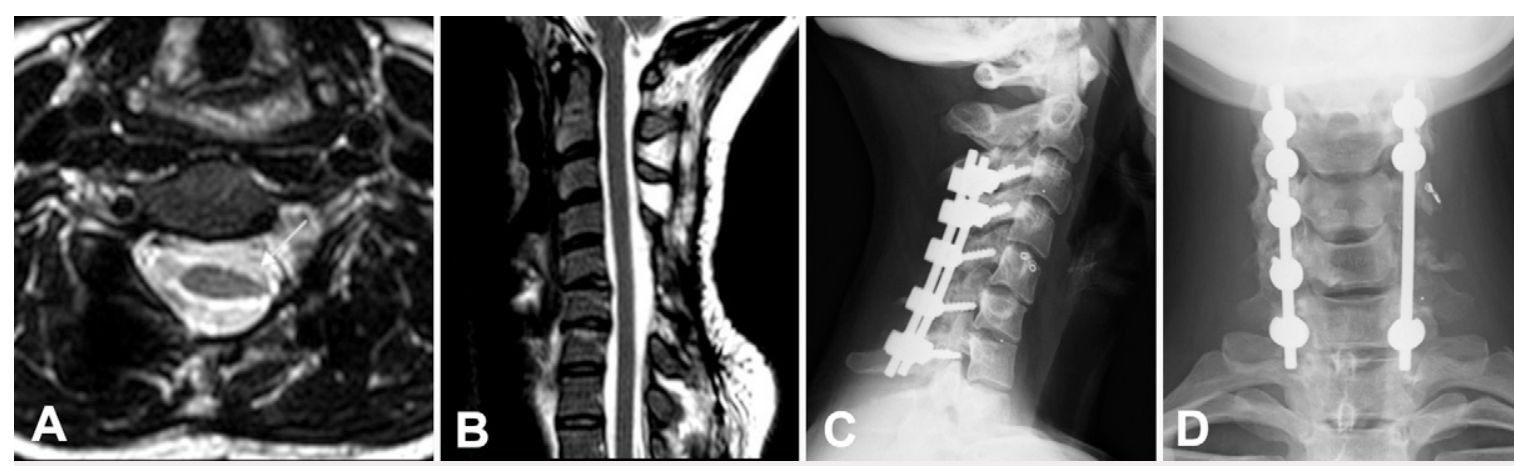

FIG. 4. A and B: Axial and sagittal fast spin-echo T2Wis. Postsurgical changes with C5-6 laminectomy, C4 laminotomy, and C3-7 arthrodesis, showing complete tumor removal ( $\mathbf{A}$ and $\mathbf{B}$ ) but discrete residual myelopathy (white arrow in $\mathbf{A}$ ). $\mathbf{C}$ and $\mathbf{D}$ : Radiography of the cervical spine. Anteroposterior and lateral view: $\mathrm{C} 3-7$ arthrodesis with posterior transpedicular screw fixation, with satisfactory bone fusion of the right posterior elements from $\mathrm{C} 4$ to $\mathrm{C} 6$. 

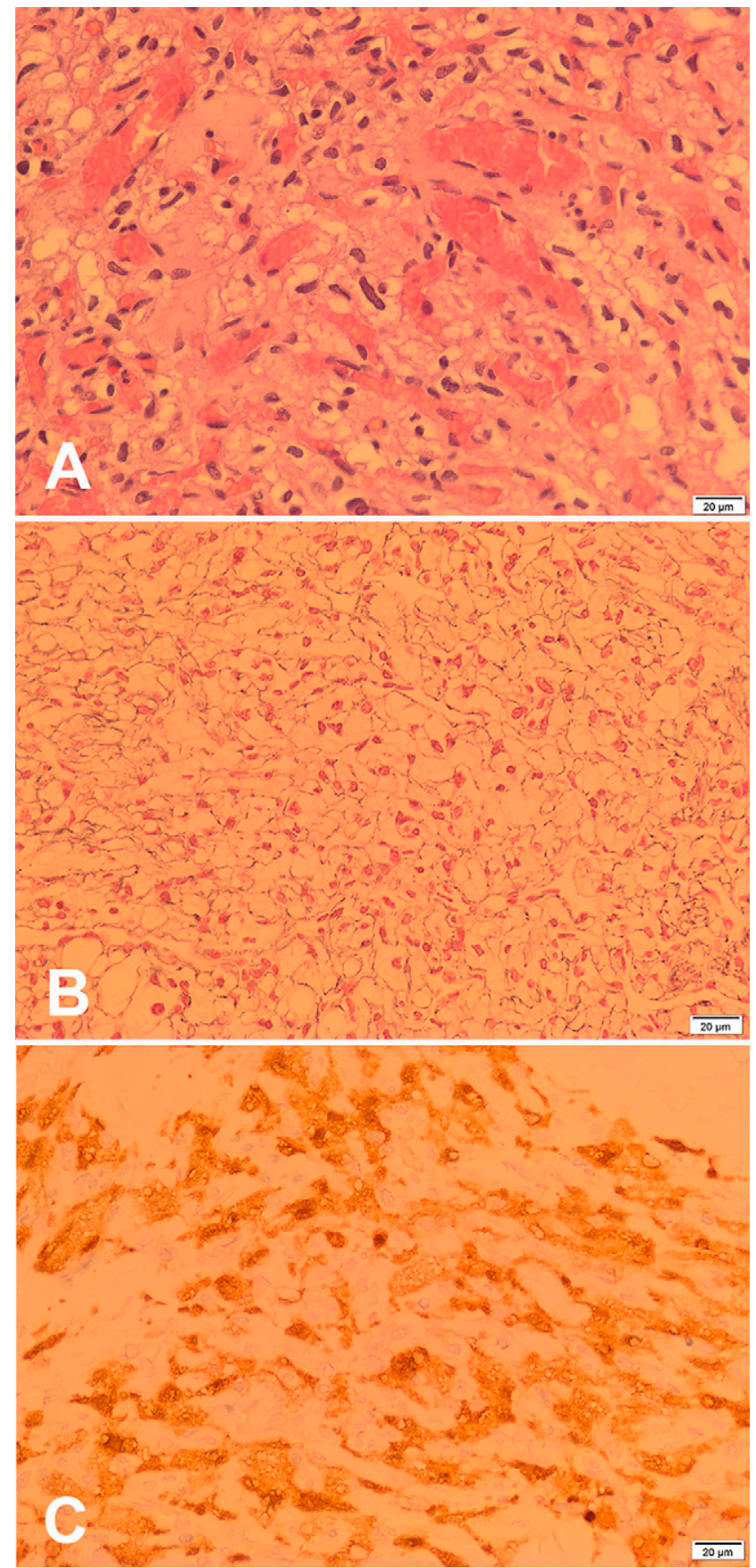

FIG. 5. Representative pathologic images. A: Hematoxylin and eosin stain. Clusters of stromal cells separated by multiple vascular channels. B: Reticulin staining readily delineates the complex capillary networks. C: Reticulin stain. Immunoreactivity for $\mathbf{S} 100$ protein is intense in the stromal cells. Bars $=20 \mu \mathrm{m}$.

facet combined with the corresponding laminectomies permitted a better exposure of the tumor and to be able to achieve an en bloc resection, with minimal spinal cord manipulation. Because the preexisting cervical spine stability was additionally compromised during the approach, instrumented fusion was required.

Unlike peripheral nerve or extraneural tissue $\mathrm{HBs}$, CNSHBs tend to have a significant cystic portion. ${ }^{14}$ Microscopic characteristics show a prominence of the stromal cell components with variable immunohistochemical expression to S-100 protein, GFAP, and vimentin. ${ }^{12}$ Sometimes, they exhibit significant nuclear pleomorphism, mimicking carcinomas or other malignant tumors. VHL gene alterations have been found in extraneural HBs, incorporating some novel mutations. ${ }^{15}$

\section{Lessons}

In this report, we highlight some of the problems encountered in the differential diagnosis and surgical management of these defying tumors involving the cervical spine. Since it can be easily mistaken for other more common types of neoplasms, it is beneficial for clinicians to acknowledge the possibility of an extradural cervical spine $\mathrm{HB}$, particularly when a rich peritumoral vascular patter is observed on MRI. In these subsets of patients, it is essential to incorporate an angio-MRI and/or angio-computed tomography in the preoperative assessment. If the suspicion of a hypervascular atypical tumor raises, a selective preoperative angiography is strongly recommended in order to demonstrate the relations of the cervical spine blood vessels (including the VA) with the tumor, which is crucial for surgical planning. Besides, it provides an opportunity to perform preoperative endovascular embolization, which is considerably convenient in these group of $\mathrm{HBs}$, whose vascular supply is known to be predominant in their extradural rather than in their intradural portion, especially in the cervical region (in proximity to vertebral and cervical spine arteries). Ignoring and skipping this procedure can lead to uncontrolled and cataclysmic bleeding, with interruption of surgery, a situation described in the past. ${ }^{4}$ Ultimately, adequate surgical exposure is mandatory to attain a safe en bloc tumor removal using precise microsurgical techniques, complemented by spinal fixation and fusion if needed.

\section{References}

1. Sid-Ahmed S, Seizeur R, Forlodou P, et al. Dumbell hemangioblastoma of the eighth cervical nerve root. Article in French. Neurochirurgie. 2006;52(6):533-536.

2. Mitchell A, Scheithauer BW, Wharen RE, Franck J, Chan K. Hemangioblastoma of spinal nerve: a report of six cases. Clin Neuropathol. 2013;32(2):91-99.

3. Zhao Y, Jin X, Gong X, et al. Clinicopathologic features of hemangioblastomas with emphases of unusual locations. Int J Clin Exp Pathol. 2017;2(10):1792-1800.

4. Aytar MH, Yener U, Ekşi MŞ, et al. Purely extradural spinal nerve root hemangioblastomas. J Craniovertebr Junction Spine. 2016;7(4): 197-200.

5. Escott EJ, Kleinschmidt-DeMasters BK, Brega K, Lillehei KO. Proximal nerve root spinal hemangioblastomas: presentation of three cases, MR appearance, and literature review. Surg Neurol. 2004;61(3):262-273.

6. Gläsker S, Berlis A, Pagenstecher A, Vougioukas VI, Van Velthoven $V$. Characterization of hemangioblastomas of spinal nerves. Neurosurgery. 2005;56(3):503-509.

7. Konovalov NA, Shishkina LV, Asyutin DS, et al. Extradural spinal cord hemangioblastoma (a case report and literature review). $\mathrm{Zh}$ Vopr Neirokhir Im N Burdenko. 2016;80(6):88-92.

8. Murota T, Symon L. Surgical management of hemangioblastoma of the spinal cord: a report of 18 cases. Neurosurgery. 1989;25(5): 699-708.

9. Doyle LA, Fletcher CDM. Peripheral hemangioblastoma: clinicopathologic characterization in a series of 22 cases. Am J Surg Pathol. 2014;38(1):119-127.

10. McCormick PC. Surgical management of dumbbell tumors of the cervical spine. Neurosurgery. 1996;38(2):294-300. 
11. Barrey C, Kalamarides M, Polivka M, George B. Cervical dumbbell intra-extradural hemangioblastoma: total removal through the lateral approach: technical case report. Neurosurgery. 2005;56(3):E625-E625.

12. Lee JW, Cho EY, Hong SH, et al. Spinal epidural hemangiomas: various types of MR imaging features with histopathologic correlation. AJNR Am J Neuroradiol. 2007;28(7):1242-1248.

13. Kim $\mathrm{CH}$, Chung $\mathrm{CK}$. Surgical outcome of a posterior approach for large ventral intradural extramedullary spinal cord tumors. Spine (Phila Pa 1976). 2011;36(8):E531-E537.

14. Hussein MR. Central nervous system capillary haemangioblastoma: the pathologist's viewpoint. Int J Exp Pathol. 2007;88(5):311-324.

15. Muscarella LA, Bisceglia M, Galliani CA, et al. Extraneuraxial hemangioblastoma: a clinicopathologic study of 10 cases with molecular analysis of the VHL gene. Pathol Res Pract. 2018;214(8):1156-1165.

\section{Disclosures}

The authors report no conflict of interest concerning the materials or methods used in this study or the findings specified in this paper.

\section{Author Contributions}

Conception and design: Piquer-Belloch, Llácer-Ortega, Llopis-San Juan. Acquisition of data: Piquer-Belloch, Rodriguez-Mena, Llácer-Ortega, Riesgo-Suárez, Cremades-Mira. Analysis and interpretation of data: Piquer-Belloch, Rodriguez-Mena, Llácer-Ortega. Drafting the article: Piquer-Belloch, Rodriguez-Mena, Llácer-Ortega, Cremades-Mira. Critically revising the article: Piquer-Belloch, Rodriguez-Mena, LlácerOrtega, Riesgo-Suárez, Cremades-Mira. Reviewed submitted version of manuscript: Piquer-Belloch, Rodriguez-Mena, Llácer-Ortega, RoviraLillo. Approved the final version of the manuscript on behalf of all authors: Piquer-Belloch. Administrative/technical/material support: Piquer-Belloch, Rodriguez-Mena, Cremades-Mira. Study supervision: Piquer-Belloch. Conducted literature search: Flor-Goikoetxea Gamo.

\section{Correspondence}

José Piquer-Belloch: Hospital Universitario de la Ribera, Valencia, Spain. piquer_jos@gva.es. 